\title{
Nonlinear Causal Cardiorespiratory Interaction in Response to Color Stimuli
}

\author{
Saman Parvaneh ${ }^{1,2}$, Sadaf Moharreri ${ }^{3}$, Shahab Rezaei ${ }^{4}$, Nader Jafarnia Dabanloo ${ }^{1}$ \\ ${ }^{1}$ Islamic Azad University, Science and Research Branch, Tehran, Iran \\ ${ }^{2}$ Philips Research North America, Cambridge, MA, USA \\ ${ }^{3}$ Islamic Azad University, Khomeini Shahr Branch, Isfahan, Iran \\ ${ }^{4}$ Islamic Azad University, Central Tehran Branch, Tehran, Iran
}

\begin{abstract}
Convergence Cross Mapping (CCM) is a nonlinear method for evaluating causality between time series. The cardiorespiratory interaction between heart rate (HR) and respiration rate (RR) time series in response to different colored lights was studied in this paper. Using ECG and belt-based respiration data for 16 female volunteers (Age: $24.75 \pm 1.43)$ recorded during eyes-closed condition (control), and while exposed to four different colored lights (red, green, blue, and yellow), CCM $M_{H R} \rightarrow R R$ and $C C M_{R R \rightarrow H R}$ were calculated. The results suggest that cardiorespiratory interaction ( $H R \rightarrow R R$ and $R R \rightarrow H R$ ) captured by CCM are different in response to four colored visual stimuli. However, statistical analysis of data revealed no statistically significant difference in these parameters $(p>0.05)$ in response to different colored light.
\end{abstract}

\section{Introduction}

Convergent Cross Mapping (CCM) is a nonlinear method for studying causality between time series [1] and is shown to be effective in quantification of nonlinear directed interaction between biomedical time series for estimating cerebral autoregulation [2] and investigating interaction between heart rate variability (HRV) and EEG before, during, and after seizure [3].

It has been shown that physiological response such as heart rate (HR), HRV, and heart rate asymmetry (HRA) can change in response to colored visual stimuli [4-6]. Furthermore, Edelhauser et al. using heart respiration ratio and phase coordination ratio showed that non-standardized red and blue lights were able to alter cardiorespiratory coordination [7]. However, little is known about how directed cardiorespiratory interaction will change in response to different colored light. This paper aims at studying nonlinear directed cardiorespiratory interaction in response to colored stimuli using CCM.

\section{Data and Method}

\subsection{Data}

Lead II of ECG and belt-based respiration (sampling frequency $=200 \mathrm{~Hz}$ ) were recorded from 16 female volunteers (Age: $24.75 \pm 1.43$ ) during eyes-closed condition (control) and while they were exposed to four different colored lights (e.g., red, green, blue, and yellow).

Duration of recording in response to each color as visual stimulus was five minutes and colors were displayed on a computer screen with a random order. A 10 minutes break was given between each color stimuli to cancel the effects of previous stimulation. Recording setting is shown in Figure 1.

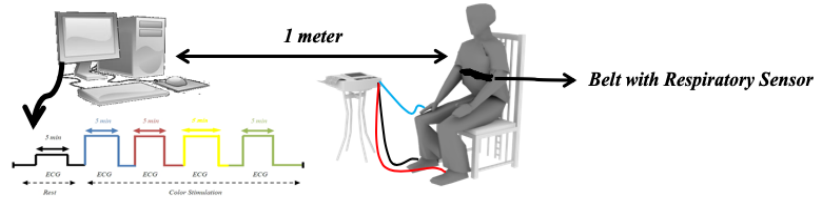

Figure 1. Recording setting for displaying different colored lights on a computer screen and simultaneous recording of ECG and belt-based respiration.

\subsection{Method}

\subsubsection{Pre-processing}

QRS peaks were detected using the Pan-Tompkins algorithm [8]. As suggested in the guideline [9], HR time series was created after manual inspection of peaks detected by the QRS detection algorithm. Furthermore, slope-based peak detection was used to find respiratory intervals and respiration rate (RR) time series from recorded belt-based respiration data. Typical ECG and respiration waveform with detected peaks are shown in Figure 2.

For causality analysis, spline interpolation from beat-tobeat signals (e.g., heart rate and respiration rate time series) was used to create an evenly sampled signal with a 
sampling frequency of $10 \mathrm{~Hz}$.

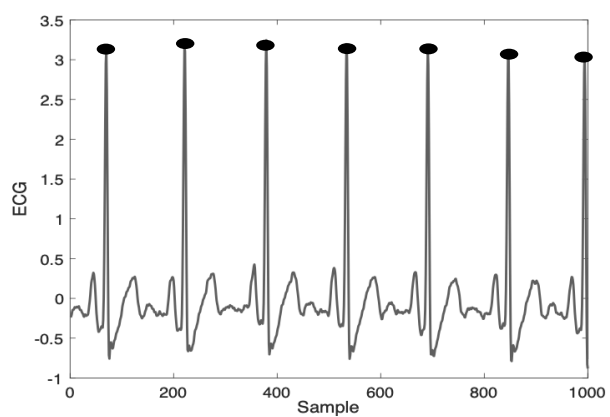

(a)

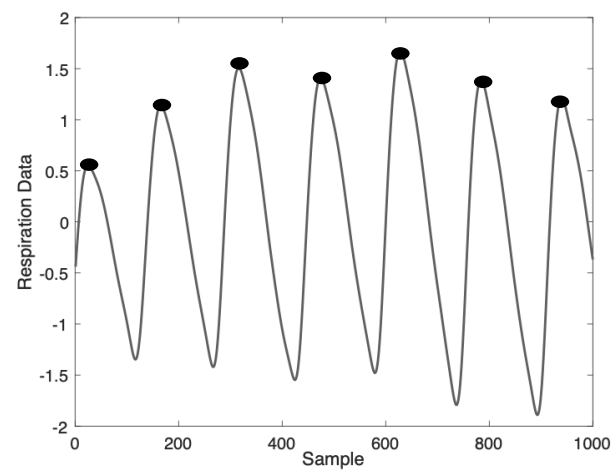

(b)

Figure 2. Typical ECG and respiration waveform with detected peaks marked with dots.

\subsubsection{Correlation}

Pearson's correlation coefficient was calculated to determine the association between heart rate and respiration rate time series. Of note, the correlation could not be used to study causation between time series.

\subsubsection{Convergent Cross Mapping}

Sugihara et al. proposed CCM as a new nonlinear method to determine causality between variables in a dynamical system [1].

In general, there would be some correspondence between shadow manifolds (reconstructed phase space) if two time series are from the same n-dimensional manifold. The extent to which the historical record of time series $\mathrm{Y}$ can estimate the states of time series $X$ (cross mapping of $\mathrm{X}$ by using $\mathrm{M}_{\mathrm{Y}}: \mathrm{X} \mid \mathrm{M}_{\mathrm{Y}}$ ) or vice versa (cross mapping of $\mathrm{Y}$ by using $\mathrm{M}_{\mathrm{X}}: \mathrm{Y} \mid \mathrm{M}_{\mathrm{X}}$ ) is measured by CCM. Strength of interaction is quantified by finding the correlation between original time series and reconstructed time series created using cross mapping:

$$
\begin{aligned}
\operatorname{CCM}_{X \rightarrow Y} & =\left|\rho\left(X, \hat{X} \mid M_{Y}\right)\right| \\
\operatorname{CCM}_{Y \rightarrow X} & =\left|\rho\left(Y, \hat{Y} \mid M_{X}\right)\right|
\end{aligned}
$$

Please to refer to reference [1] for details on CCM estimation.
For creating shadow manifold, the optimal value for the embedding dimension was estimated using the false nearest neighbor (FNN) method [10], and the optimum delay was chosen empirically. MATLAB (MathWorks, Natick, MA) was used for pre-processing and estimation of correlation and CCM.

\subsubsection{Statistical Analysis}

Mean and standard deviation were reported for correlation and CCM between heart rate and respiration rate time series in response to different colors. Furthermore, to study the association between HR and RR and nonlinear causal interaction from $\mathrm{HR}$ to $\mathrm{RR}$ $\left(\mathrm{CCM}_{\mathrm{HR} \rightarrow \mathrm{RR}}\right)$ and from RR to $\mathrm{HR}\left(\mathrm{CCM}_{\mathrm{RR} \rightarrow \mathrm{HR}}\right)$, correlation and causal interaction for exposure to different colored light were compared using Friedman test. The statistical significance level was set to 0.05 . All statistical analysis was done in SPSS (IBM, version 25, Chicago, IL).

\section{Results and Discussion}

Mean and standard deviation of correlation between HR

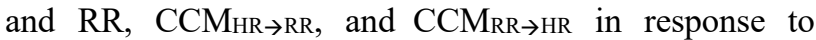
different colored lights are reported in Table 1. Furthermore, the changes in these parameters across groups with different color stimuli are visually depicted in Figure 3. For CCM analysis, the optimal value for embedding dimension was found equal to four using FNN and delay of one was chosen empirically.

Despite a decreasing trend in correlation between HR and RR, $\mathrm{CCM}_{\mathrm{HR} \rightarrow \mathrm{RR}}$, and $\mathrm{CCM}_{\mathrm{RR} \rightarrow \mathrm{HR}}$ from control to stimulation by green, yellow, blue, and red, there were no significant differences in these parameters in control condition and in response to different colored light. More specifically, Friedman test revealed no statistically significant difference in correlation between $\mathrm{HR}$ and $\mathrm{RR}$ $(p=0.462), \quad C C M_{H R \rightarrow R R} \quad(p=0.272), \quad$ and $\quad C_{C M R} \rightarrow H R$ $(\mathrm{p}=0.105)$ in response to different colored light was used as visual stimuli. To better visualize the distribution of correlation between $\mathrm{HR}$ and $\mathrm{RR}, \mathrm{CCM}_{\mathrm{HR} \rightarrow \mathrm{RR}}$, and $\mathrm{CCM}_{\mathrm{RR} \rightarrow \mathrm{HR}}$, boxplots of these parameters across groups with different colored light stimuli is shown in Figure 4.

Table 1. Mean and standard deviation of correlation between $\mathrm{HR}$ and RR, $\mathrm{CCM}_{\mathrm{HR} \rightarrow \mathrm{RR}}$, and $\mathrm{CCM}_{\mathrm{RR} \rightarrow \mathrm{HR}}$ across groups with different colored light stimuli (CCM: Convergence Cross Mapping)

\begin{tabular}{cccc} 
& Correlation & $\mathrm{CCM}_{\mathrm{HR} \rightarrow \mathrm{RR}}$ & $\mathrm{CCM}_{\mathrm{RR} \rightarrow \mathrm{HR}}$ \\
\hline Red & $0.486 \pm 0.295$ & $0.459 \pm 0.316$ & $0.494 \pm 0.310$ \\
\hline Blue & $0.552 \pm 0.283$ & $0.555 \pm 0.321$ & $0.481 \pm 0.352$ \\
\hline Yellow & $0.546 \pm 0.278$ & $0.545 \pm 0.292$ & $0.547 \pm 0.322$ \\
\hline Green & $0.587 \pm 0.309$ & $0.612 \pm 0.326$ & $0.590 \pm 0.332$ \\
\hline Control & $0.680 \pm 0.273$ & $0.687 \pm 0.297$ & $0.577 \pm 0.333$ \\
\hline
\end{tabular}




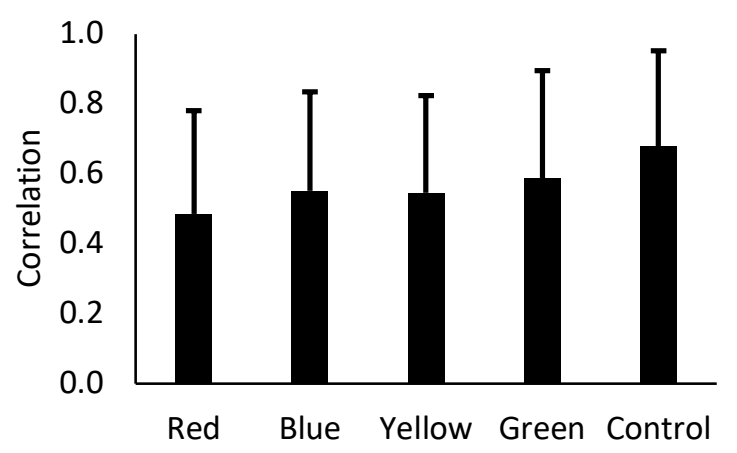

(a)

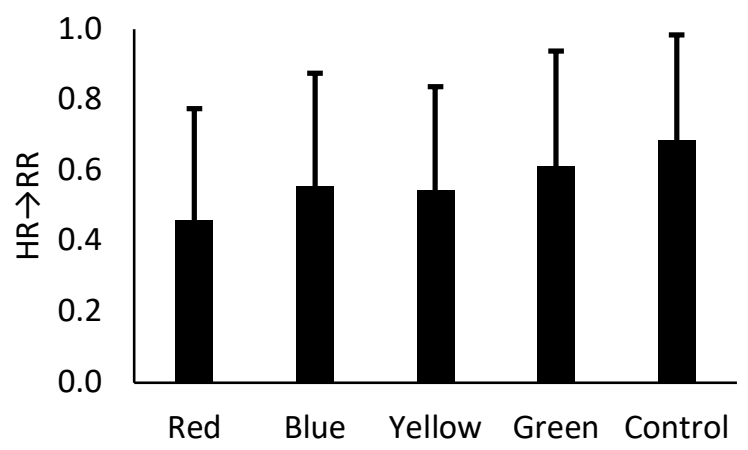

(b)

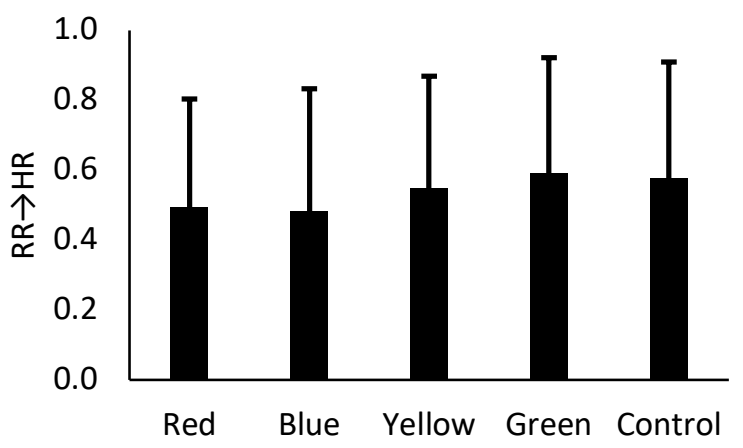

(c)

Figure 3. (a) Correlation between $\mathrm{HR}$ and RR, (b) $\mathrm{CCM}_{H R \rightarrow R R}$, and (c) $\mathrm{CCM}_{\mathrm{RR} \rightarrow \mathrm{HR}}$ for groups with different colored light stimuli (mean values and standard deviations are presented).

$\mathrm{CCM} \mathrm{MR}_{\mathrm{H}} \rightarrow \mathrm{RR}$ and $\mathrm{CCM} \mathrm{RR}_{\mathrm{R}} \rightarrow \mathrm{HR}$ during the control condition and in response to different colors are shown in Figure 5. Non-zero values for these parameters show a bidirectional interaction between heart rate and respiration rate in response to colored lights. The causality strength from $\mathrm{HR}$ to RR was higher in the control condition as well as in response to blue and green compared to causality strength from RR to HR. However, causality strength from RR to HR was slightly higher in response to red and yellow as color stimuli compared to causality strength from HR to RR. The results highlight the potential of nonlinear causality analysis to detect changes in cardiorespiratory interaction in response to color stimuli.

Previous research confirmed changes in $\operatorname{HRV}[5,6,11$, 12] and cardiorespiratory coordination [7] in response to colored light. This study is the first study that studied the changes in nonlinear directed interaction in response to external colored lights.

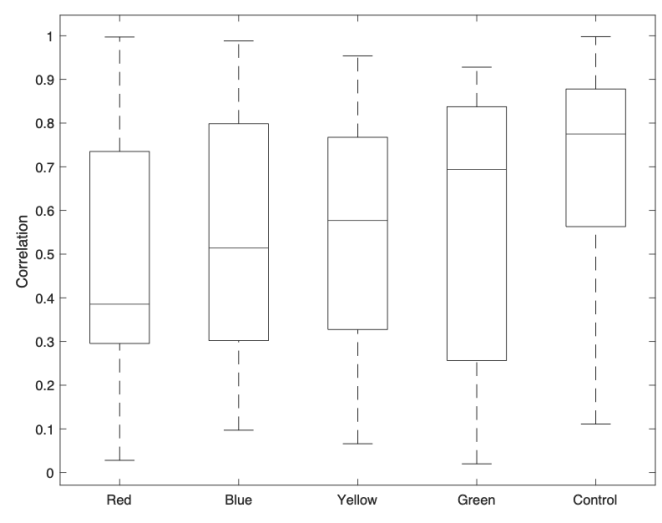

(a)

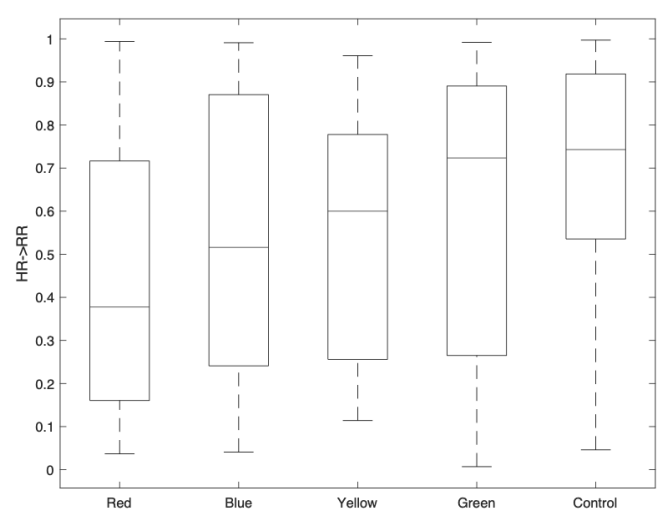

(b)

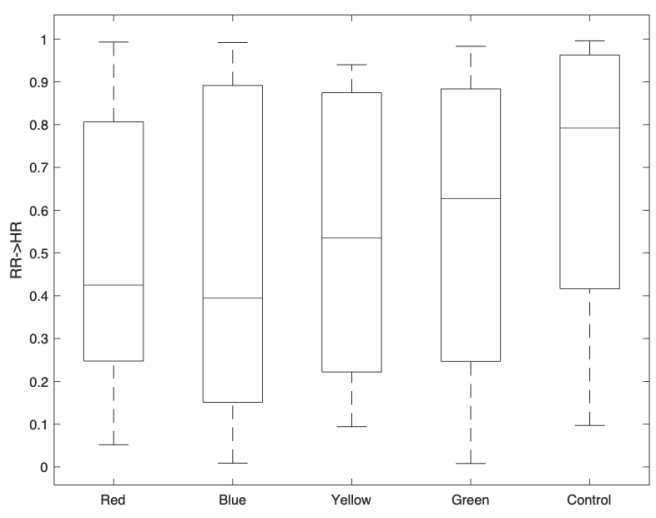

(c)

Figure 4. Boxplot representation of (a) correlation between $\mathrm{HR}$ and RR, (b) $\mathrm{CCM}_{\mathrm{HR} \rightarrow \mathrm{RR}}$, and (c) $\mathrm{CCM}_{\mathrm{RR} \rightarrow \mathrm{HR}}$ in response to different colored lights. 


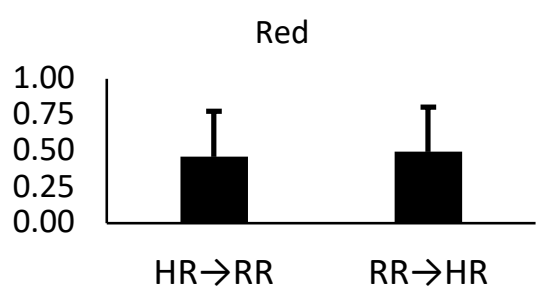

(a)



(b)

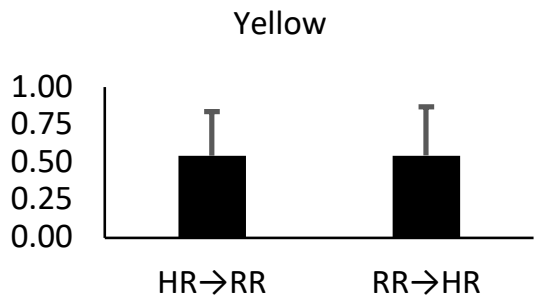

(c)

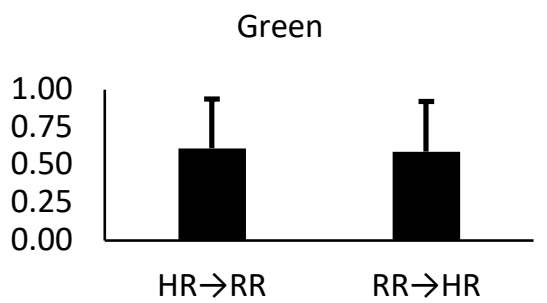

(d)

Control

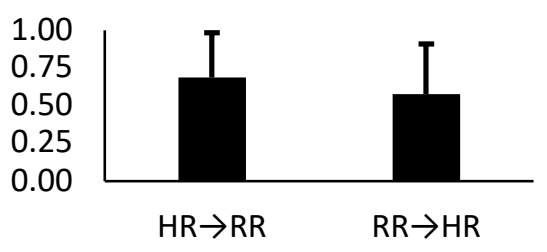

(e)

Figure 5. $\mathrm{CCM}_{\mathrm{HR} \rightarrow \mathrm{RR}}$ and $\mathrm{CCM}_{\mathrm{RR} \rightarrow \mathrm{HR}}$ in response to (a) red, (b) blue, (c) yellow, (d) green, and (e) control (mean values and standard deviations are presented).

\section{Conclusion}

In this article, convergence cross mapping was used to study nonlinear directed interaction between heart rate and respiration time series, cardiorespiratory interaction, across groups with different colored light stimuli. The results suggest a bidirectional interaction between heart rate and respiration rate in response to colored lights and highlight the potential of nonlinear directed causality analysis to detect changes in cardiorespiratory interaction in response to color stimuli.

\section{References}

[1] G. Sugihara et al., "Detecting causality in complex ecosystems," science, vol. 338, no. 6106, pp. 496-500, 2012.

[2] L. Heskamp, A. Meel-van den Abeelen, J. Lagro, and J. Claassen, "Convergent cross mapping: a promising technique for cerebral autoregulation estimation," Int. J. Clin. Neurosci. Ment. Heal. S, vol. 20, 2014.

[3] K. Schiecke et al., "Nonlinear directed interactions between HRV and EEG activity in children with TLE," IEEE Transactions on Biomedical Engineering, vol. 63, no. 12, pp. 2497-2504, 2016.

[4] L. Laufer, E. Lang, L. Izso, and E. Nemeth, "Psychophysiological effects of coloured lighting on older adults," Lighting Research \& Technology, vol. 41, no. 4, pp. 371-378, 2009.

[5] S. Moharreri, N. J. Dabanloo, S. Parvaneh, and A. M. Nasrabadi, "How to Interpret Psychology from Heart Rate Variability," Middle East Conference on Biomedical Engineering, 2011.

[6] S. Parvaneh, N. J. Dabanloo, S. Rezaei, S. Moharreri, and $\mathrm{N}$. Toosizadeh, "Heart rate asymmetry in response to colored light," in 2017 Computing in Cardiology (CinC), 2017: IEEE, pp. 1-4.

[7] F. Edelhäuser et al., "Impact of colored light on cardiorespiratory coordination," Evidence-Based Complementary and Alternative Medicine, vol. 2013, 2013.

[8] J. Pan and W. J. Tompkins, "A real-time QRS detection algorithm," Biomedical Engineering, IEEE Transactions on, no. 3, pp. 230-236, 1985.

[9] M. Malik, "Heart rate variability," Annals of Noninvasive Electrocardiology, vol. 1, no. 2, pp. 151-181, 1996.

[10] M. B. Kennel, R. Brown, and H. D. Abarbanel, "Determining embedding dimension for phase-space reconstruction using a geometrical construction," Physical review A, vol. 45, no. 6, p. 3403, 1992.

[11] S. Moharreri, S. Rezaei, N. J. Dabanloo, and S. Parvaneh, "Study of induced emotion by color stimuli: power spectrum analysis of heart rate variability," in Computing in Cardiology, 2014.

[12] A. Schäfer and K. W. Kratky, "The effect of colored illumination on heart rate variability," Complementary Medicine Research, vol. 13, no. 3, pp. 167-173, 2006.

Address for correspondence.

Saman Parvaneh

2 Canal Park, 3rd floor, Cambridge, MA 02141

parvaneh@ieee.org 